\title{
Neural processing of repetition and non-repetition grammars in 7- and 9-month-old infants
}

\author{
Jennifer B. Wagner ${ }^{1}$, Sharon E. Fox ${ }^{1,2}$, Helen Tager-Flusberg ${ }^{3}$ and Charles A. Nelson ${ }^{1}$ \\ 1 Laboratories of Cognitive Neuroscience, Division of Developmental Medicine, Children's Hospital Boston/Harvard Medical School, Boston, MA, USA \\ 2 MIT Health Sciences and Technology, Cambridge, MA, USA \\ ${ }^{3}$ Department of Psychology, Boston University, Boston, MA, USA
}

\author{
Edited by: \\ Judit Gervain, CNRS-Université Paris \\ Descartes, France \\ Reviewed by: \\ Judit Gervain, CNRS-Université Paris \\ Descartes, France \\ Hugh Rabagliati, Brown University, \\ USA

\section{*Correspondence:} \\ Jennifer B. Wagner, Laboratories of \\ Cognitive Neuroscience, Division of \\ Developmental Medicine, Children's \\ Hospital Boston, 1 Autumn Street, \\ AU641, Boston, MA 02215, USA. \\ e-mail: jen.wagner@alum.mit.edu
}

An essential aspect of infant language development involves the extraction of meaningful information from a continuous stream of auditory input. Studies have identified early abilities to differentiate auditory input along various dimensions, including the presence or absence of structural regularities. In newborn infants, frontal and temporal regions were found to respond differentially to these regularities (Gervain et al., 2008), and in order to examine the development of this abstract rule learning we presented 7- and 9-month-old infants with syllables containing an ABB pattern (e.g., "balolo") or an ABC pattern (e.g., "baloti") and measured activity in left and right lateral brain regions using near-infrared spectroscopy (NIRS). While prior newborn work found increases in oxyhemoglobin (oxyHb) activity in response to ABB blocks as compared to $\mathrm{ABC}$ blocks in anterior regions, 7- and 9-month-olds showed no differentiation between grammars in oxy $\mathrm{Hb}$. However, changes in deoxyhemoglobin (deoxyHb) pointed to a developmental shift, whereby 7-month-olds showed deoxy Hb responding significantly different from zero for $\mathrm{ABB}$ blocks, but not $\mathrm{ABC}$ blocks, and 9-month-olds showed the opposite pattern, with deoxyHb responding significantly different from zero for the $A B C$ blocks but not the $A B B$ blocks. DeoxyHb responses were more pronounced over anterior regions. A grammar by time interaction also illustrated that during the early blocks, deoxyHb was significantly greater to $A B C$ than in later blocks, but there was no change in $A B B$ activation over time. The shift from stronger activation to ABB in newborns (Gervain et al., 2008) and 7-month-olds in the present study to stronger activation to $A B C$ by 9-month-olds here is discussed in terms of changes in stimulus salience and novelty preference over the first year of life. The present discussion also highlights the importance of future work exploring the coupling between oxyHb and deoxy $\mathrm{Hb}$ activation in infant NIRS studies.

Keywords: infancy, auditory processing, NIRS, optical imaging, language

\section{INTRODUCTION}

The first year of life represents an important period for language development that culminates with infants producing their first words. Even before they begin speaking, however, research has shown that infants can pick up on important and complex linguistic cues from their auditory input, including rhythmical cues (Mehler et al., 1988), phonemic contrasts (e.g., Werker and Tees, 1984; Kuhl et al., 2006), structural regularities (e.g., Christophe et al., 1994), and transitional probabilities (e.g., Saffran et al., 1996; Teinonen et al., 2009; for a review of early infant language development, see Gervain and Mehler, 2010).

A rich set of behavioral work with infants during the first year of life has highlighted abilities for extracting rules from stimuli varying in repetition structure. Seminal work by Marcus et al. (1999) found that 7-month-old infants tracked an embedded syllable structure and later used this to generalize to novel stimuli. Subsequent work has focused on the generalizability of this rule-learning mechanism, including the ability to extract rule information when syllables are substituted with other auditory sounds, such as tones (e.g., Marcus et al., 2007; Dawson and Gerken, 2009), the use of redundant cues to support rule learning (e.g., Frank et al., 2009), changing stimulus salience over development (e.g., Dawson and Gerken, 2009), and rule abstraction across visual domains (Saffran et al., 2007; Johnson et al., 2009). The behavioral literature points to shifting rule abstraction abilities between 5 - and 12 -months, but little work has examined the neural correlates of pattern detection abilities in this age group.

A growing set of infant language studies have utilized nearinfrared spectroscopy (NIRS), an optical imaging method that assesses hemodynamic response in awake participants, to examine the brain regions activated during processing and discrimination of linguistically relevant auditory input within the first few months of life (e.g., Pena et al., 2003; Homae et al., 2006; Saito et al., 2007a,b; Gervain et al., 2008; Telkemeyer et al., 2009; see Obrig et al., 2010; Gervain et al., 2011, for reviews). For example, Pena et al. (2003) presented newborn infants with blocks of forward and backward speech and used NIRS to measure hemodynamic response in the left and right hemisphere during these auditory streams. During forward speech, Pena et al. (2003) found greater activation in left temporal areas. More specifically relating to the rule-learning literature, Gervain et al. (2008) also examined auditory processing in newborn infants using NIRS, presenting blocks of trisyllabic words 
with either an $\mathrm{ABB}$ pattern or an $\mathrm{ABC}$ pattern. Newborns showed greater activation to the $\mathrm{ABB}$ grammar than the $\mathrm{ABC}$ grammar, providing evidence that infants are capable of extracting regularities from their speech stream from birth (Gervain et al., 2008).

Although NIRS has provided researchers with the opportunity to examine the neural bases of auditory processing, and in the case of Gervain et al. (2008), repetition detection in very young infants, few studies thus far have used this novel method to study auditory processing in infants beyond 6-months-of-age (e.g., Homae et al., 2007; Minagawa-Kawai et al., 2007; Sato et al., 2009; see Obrig et al., 2010, for a review). By looking at a single auditory processing task over a wider range of ages, researchers can develop a more comprehensive understanding of how auditory and linguistic processing change over the course of infancy. Work by Minagawa-Kawai et al. (2007) adopted this developmental approach and used NIRS to examine the discrimination of phonemic contrasts in infants ranging from 3- to 28-months-of-age. Minagawa-Kawai et al. (2007) identified a developmental shift in oxyhemoglobin responses to across-category and within-category phonemic contrasts, as well as changes in hemispheric lateralization over the first 2 years of life.

The present study aimed to examine the neural basis of linguistic processing of repetition- and non-repetition-based grammars during the first year of life, extending from the paradigm developed by Gervain et al. (2008). With a host of behavioral work showing subtle shifts in development with respect to abstracting rules from input, this work will contribute to a neural framework of learning and pattern extraction. Seven- and 9-month-old infants were presented with grammars that did or did not contain syllables with a repetition (ABB vs. ABC), and NIRS was used to capture hemodynamic changes throughout the task. This work aimed to provide a richer picture of the neural correlates of auditory processing and pattern detection, across the first year of life.

\section{MATERIALS AND METHODS PARTICIPANTS}

The final sample consisted of thirteen 7-month-old infants (mean age $=208$ days, $\mathrm{SD}=17$; six female infants) and fifteen 9-month-old infants (mean age $=286$ days, $\mathrm{SD}=11$; nine female infants). An additional five 7-month-old infants and two 9-month-old infants participated in the experiment, but were excluded due to excessive noise due to hair and/or motion artifact that resulted in missing data for over $30 \%$ of channels. All infants included in the experiment were: (1) born after 36 weeks gestational age, (2) born weighing more than $2500 \mathrm{~g}$, and (3) born without a known neurological abnormality. Project approval was obtained from the Institutional Review Board of Children's Hospital Boston. Written informed consent was obtained from the parents of all infant participants.

\section{STIMULI}

Stimuli consisted of trisyllabic sequences identical to those used in a prior study by Gervain et al. (2008) with newborn infants that presented a repetition based $\mathrm{ABB}$ artificial grammar (e.g., "balolo") and an unstructured ABC control grammar (e.g., "baloti"; see Gervain et al. for further details of the $\mathrm{ABB}$ and $\mathrm{ABC}$ grammar construction). $\mathrm{ABB}$ and $\mathrm{ABC}$ grammars were matched in syllabic repertoire, frequency of syllables, and transitional probabilities between syllables.
Trisyllabic sequences were grouped into blocks of 10 , with intervals of $0.5-1.5 \mathrm{~s}$ of silence occurring between each sequence. Each block was, on average, $16 \mathrm{~s}$ in length. Blocks of the ABB and $\mathrm{ABC}$ grammars were presented in one of two semi-randomized sequences, and were separated by a silent pause of varying duration (15 s minimum).

\section{APPARATUS}

A Hitachi ETG-4000 NIRS system with 24 simultaneously recording channels was used to collect hemodynamic response during stimulus presentation. Two wavelengths of light (695 and $830 \mathrm{~nm}$ ) were used to measure cortical levels of oxyhemoglobin and deoxyhemoglobin (oxyHb and deoxyHb). The near-infrared light was guided by optical fiber bundles that were $1 \mathrm{~mm}$ in diameter. On the ETG-4000 device, each pair of adjacent incident and detection fibers defines a single measurement channel, allowing the measurement of the hemodynamic changes in the brain corresponding to a specific stimulus. The NIRS probes consist of two $3 \times 3$ chevron arrays, each with five emitting and four detecting fibers held in place by a silicone support with $3 \mathrm{~cm}$ spacing, and attached to a soft cap designed for infants (see Figure 1A). The flexibility of the silicone supported by an adjustable neoprene band allowed for a bilateral placement of the probes spanning from anterior to posterior lateral regions on each side of the head (see Figure 1B).

\section{PROCEDURE}

Infants were seated on a parent's lap throughout the experiment. Infants passively listened to the auditory stimuli, which were presented through speakers placed behind a curtain in front of the infants. During presentation of the $\mathrm{ABB}$ and $\mathrm{ABC}$ blocks, infants were presented with a video containing shapes moving on a screen, and when uninterested in the videos, an experimenter used silent toys and bubbles to keep infants calm and still. Infants who became fussy were permitted to nurse, feed from a bottle, or eat finger foods to expose them to as many blocks as possible. While this had the potential to introduce motion artifacts, past work recording EEG during auditory stimuli (a method much more susceptible to motion artifacts than NIRS) with infants who were nursing or bottle-feeding obtained sufficient artifact-free data for analyses under similar circumstances (Thomas and Lykins, 1995; Little et al., 1999). Generally, trials were presented until the infant heard all 28 blocks, or until the infant became too fussy to continue.

\section{DATA ANALYSIS}

Based on the light intensity detected through each channel, relative concentrations of oxyHb and deoxyHb were calculated from absorbance at each wavelength using the modified Beer-Lambert law. This conversion, as well as further data analyses, was implemented through customized Matlab scripts (version 7.6, Mathworks Inc., Natick, MA, USA).

Timeseries corresponding to oxy $\mathrm{Hb}$ and deoxy $\mathrm{Hb}$ values were first processed using a fifth order Butterworth filter between 0.01 and $1.0 \mathrm{~Hz}$, and additional artifacts were identified and extracted if the raw signal exceeded a threshold value (4.95), indicating saturation of the detector optodes, or if total hemoglobin change exceeded $0.3 \mathrm{mM}^{\star} \mathrm{mm}$ within a 0.7 -s time window. For each subject with at least 10 trials of each condition $(\mathrm{ABB}$ and $\mathrm{ABC})$ and no significant 
artifacts after initial filtering, the data from each block were parsed into a 16-s time window with $0.1 \mathrm{~s}$ time resolution beginning at stimulus onset. In addition to inter-block intervals of at least $15 \mathrm{~s}$ to allow the hemodynamic response to return to baseline, each $16 \mathrm{~s}$ time window was corrected to a zero baseline value at stimulus onset in order to allow for standardized comparisons across conditions. Specifically, for each trial, the oxyHb and deoxyHb value in the first $0.1 \mathrm{~s}$ time bin was subtracted from itself to create a zero baseline in each channel at the start of every trial. This value was then subtracted from each subsequent time point for a given channel to adjust the entire trial by this baseline correction within the initial time bin.

Each block from a single participant was grouped by grammar type and averaged to obtain mean values of oxyHb and deoxy $\mathrm{Hb}$ across time for each individual channel during the $\mathrm{ABB}$ and $\mathrm{ABC}$ blocks. On a subject-by-subject level, any channels with low oxyHb signal-to-noise (Mean/SD < 1.0) were excluded from subsequent analyses. If this analysis of noise revealed a loss of more than $30 \%$ of channels, infants were excluded from further analyses. On average, infants included in subsequent analyses lost $12 \%$ of channels due to low signal-to-noise ( $\mathrm{SD}=8 \%)$.

Based on the work of Gervain et al. (2008), two sets of analyses were conducted on the present data. The primary analyses examined the average value of oxy $\mathrm{Hb}$ and deoxy $\mathrm{Hb}$ across the 16-s time window for each grammar type at each channel. A second analysis calculated the average oxyHb and deoxy $\mathrm{Hb}$ response from the first four blocks of each condition and the last four blocks of each condition for every channel to examine differential activation across time. For both analyses, channels were grouped into four regions, Left Anterior (channels 1-5), Right Anterior (channels 20-24), Left Posterior (channels 6-12), and Right Posterior (channels 13-19). The probes were positioned such that posterior regions were posterior to the infant's ear (see Figure 1).

\section{RESULTS}

Figure 2 illustrates the grand average oxy $\mathrm{Hb}$ and deoxyHb responses to the $\mathrm{ABB}$ and $\mathrm{ABC}$ grammars for each age group across all trials, collapsed across the 24 channels used in subsequent analyses. Preliminary repeated-measures ANOVAs were run to examine the between-subjects effect of gender for each of the four analyses outlined below (average oxyHb response, average deoxyHb response, oxy $\mathrm{Hb}$ response over time, and deoxyHb response over time). No significant main effects were found and subsequent analyses collapsed across gender.

Initial analyses examined the changes in oxyHb and deoxyHb by the $\mathrm{ABB}$ and $\mathrm{ABC}$ grammars in each channel using paired $t$-tests for each age group. No single channel showed a significant difference between conditions in oxyHb or deoxyHb at either age (channelby-channel $t$-maps for oxy $\mathrm{Hb}$ and deoxyHb in 7-and 9-month-old infants are illustrated in the Appendix).

\section{AVERAGE OXYHB AND DEOXYHB RESPONSE ACROSS ALL TRIALS Average oxyHb response}

Based on the average oxyHb concentration calculated across the 16-s time window for all trials, a 2 (Grammar: ABB, ABC) $\times 2$ (Hemisphere: Left, Right) $\times 2$ (Region: Anterior, Posterior) $\times 2$ (Age: 7-month-old, 9-month-old) repeated-measures ANOVA
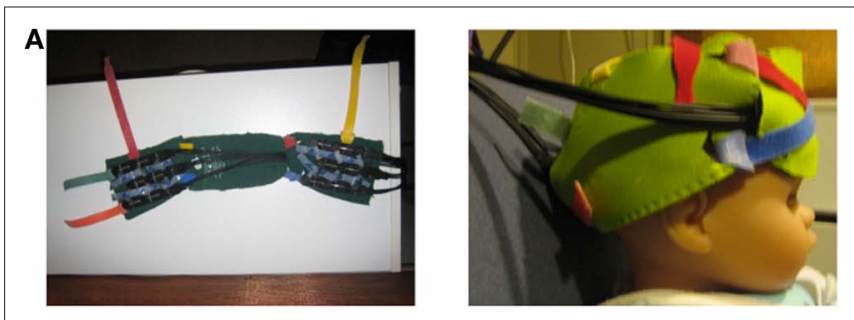

B

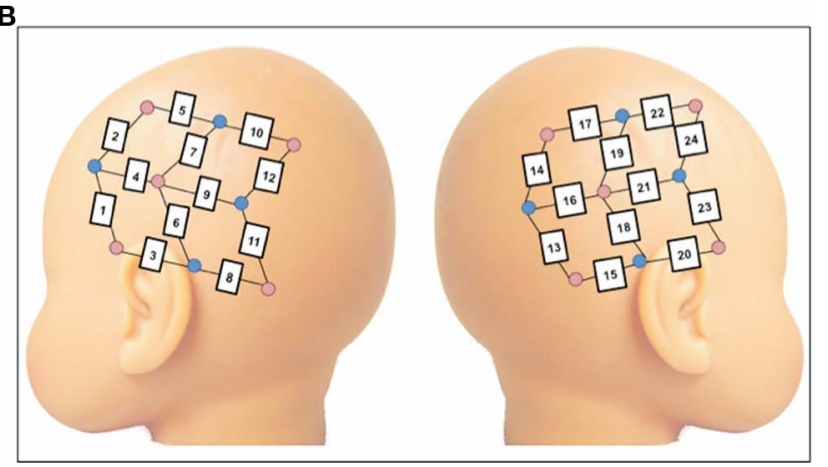

FIGURE 1 | (A) Images of the neoprene headband used to hold the NIRS probes in place in the present experiment. (B) Schematic of probe placement over the left and right lateral regions of the infant head. Due to individual variation, analyses were based on groups of channels to create standardized regions of interest across infants. The left anterior region included channels 1-5; the left posterior region included channels $6-12$, the right anterior region included channels 20-24, and the right posterior region included channels 13-19.

with grammar, hemisphere, and region as within-subjects factors and age as the between-subjects factor revealed a marginal three-way interaction between grammar, hemisphere, and region, $F(1,26)=3.29, p=0.08, \eta_{p}^{2}=0.11$, but no other significant main effects or interactions $(p s>0.12)$. This trend showed that oxyHb responses in the left hemisphere appeared similar in the anterior and posterior regions for the $\mathrm{ABB}$ grammar (ABB Left Anterior: $M=0.018 \mathrm{mmol}^{\star} \mathrm{mm}, \mathrm{SD}=0.009$; $\mathrm{ABB}$ Left Posterior: $M=0.014 \mathrm{mmol}^{*} \mathrm{~mm}, \mathrm{SD}=0.013$ ) and for the $\mathrm{ABC}$ grammar (ABC Left Anterior: $M=0.020 \mathrm{mmol}^{\star} \mathrm{mm}, \mathrm{SD}=0.010$; ABC Left Posterior: $\left.M=0.018 \mathrm{mmol}^{\star} \mathrm{mm}, \mathrm{SD}=0.014\right)$. In the right hemisphere, however, the ABB grammar trended toward larger responding in the anterior region $\left(M=0.026 \mathrm{mmol}^{\star} \mathrm{mm}, \mathrm{SD}=0.019\right)$ as compared to the posterior region $\left(M=0.005 \mathrm{mmol}^{\star} \mathrm{mm}\right.$, $\mathrm{SD}=0.015)$, while the $\mathrm{ABC}$ grammar showed the opposite pattern (ABC Right Anterior: $M=0.004 \mathrm{mmol}^{\star} \mathrm{mm}, \mathrm{SD}=0.011$; $\mathrm{ABC}$ Right Posterior: $M=0.025 \mathrm{mmol}^{\star} \mathrm{mm}, \mathrm{SD}=0.017$ ).

\section{Average deoxyHb response}

Using the average deoxyHb concentration calculated across the 16-s time window for all trials, a 2 (Grammar: $\mathrm{ABB}, \mathrm{ABC}) \times 2$ (Hemisphere: Left, Right $) \times 2$ (Region: Anterior, Posterior $) \times 2$ (Age: 7-month-old, 9-month-old) repeated-measures ANOVA with grammar, hemisphere, and region as within-subjects factors and age as the between-subjects factor revealed a main effect of region, $F(1,26)=13.19, p=0.001$, $\eta_{\mathrm{p}}^{2}=0.34$, whereby a stronger negative response was found over 



FIGURE 2 |Time course of oxy $\mathrm{Hb}$ and deoxy Hb. Illustration of the average oxyHb and deoxyHb concentration at each time point across the 16-s time window for the $\mathrm{ABB}$ and $\mathrm{ABC}$ grammar in the group of 7-month-old infants (left) and the group of 9-month-old infants (right). Concentrations are averaged across all 24 channels.

anterior regions $\left(M=-0.016 \mathrm{mmol}^{\star} \mathrm{mm}, \mathrm{SD}=0.016\right)$ as compared to posterior regions $\left(M=-0.003 \mathrm{mmol}^{*} \mathrm{~mm}, \mathrm{SD}=0.018\right)$. A significant interaction between hemisphere and region was also identified, $F(1,26)=5.71, p=0.024, \eta_{p}^{2}=0.18$. Post hoc pairwise comparisons revealed that for anterior regions, the right hemisphere showed a greater negative deoxyHb response $\left(M=-0.021 \mathrm{mmol}^{*} \mathrm{~mm}, \mathrm{SD}=0.023\right)$ as compared to the left hemisphere $\left(M=-0.012 \mathrm{mmol}^{*} \mathrm{~mm}, \mathrm{SD}=0.016\right)$, $t(27)=2.11, p=0.044, d=0.46$, but in posterior regions, there was no significant difference between hemispheres, $t(27)=-0.92, p=0.37$, $d=0.19$ (Left Posterior: $M=-0.005 \mathrm{mmol}^{\star} \mathrm{mm}, \mathrm{SD}=0.021$; Right Posterior: $\left.M=-0.001 \mathrm{mmol}^{\star} \mathrm{mm}, \mathrm{SD}=0.022\right)$.

Further, a marginal interaction was observed between grammar and age, $F(1,26)=3.18, p=0.086, \eta_{p}^{2}=0.11$, whereby 7 -month-old infants showed a greater negative deoxyHb response to the $\mathrm{ABB}$ gram$\operatorname{mar}\left(M=-0.018 \mathrm{mmol}^{\star} \mathrm{mm}, \mathrm{SD}=0.022\right)$ as compared to the $\mathrm{ABC}$ $\operatorname{grammar}\left(M=-0.005 \mathrm{mmol}^{\star} \mathrm{mm}, \mathrm{SD}=0.024\right)$, and 9-month-old infants showed the opposite pattern, with larger negative deoxyHb responding to the $\mathrm{ABC}$ grammar $\left(M=-0.014 \mathrm{mmol}^{*} \mathrm{~mm}, \mathrm{SD}=0.025\right)$ as compared to the $\mathrm{ABB}$ grammar $\left(M=-0.002 \mathrm{mmol}^{\star} \mathrm{mm}\right.$, $\mathrm{SD}=0.023$ ). Follow-up one-sample $t$-tests revealed that in 7 -montholds, the $\mathrm{ABB}$ deoxyHB response was significantly different from zero, $t(12)=-2.879, p=0.014, d=0.80$, and in 9-month-olds, the $\mathrm{ABC}$ deoxyHb response was significantly different from zero, $t(14)=-2.220, p=0.043, d=0.57$. The 7-month-old ABC deoxyHb response and the 9-month-old $\mathrm{ABB}$ deoxyHb responses were not significantly different from zero ( $p s>0.45$; Figure 3 ).

\section{AVERAGE OXYHB AND DEOXYHB RESPONSE: EARLY VS. LATE BLOCKS}

Based on the work of Gervain et al. (2008), hemodynamic activity was compared between the first four blocks of each condition and the last four blocks of each condition to examine differential activation over time. These analyses included thirteen 7-monthold-infants, and eleven 9-month-old infants. The remaining four 9-month-olds who were included in the first analysis were excluded from the response-over-time analysis because they made it through less than 21 trials before becoming fussy.

\section{OxyHb response across blocks}

A 2 (Time: First 4 blocks, Last 4 blocks) $\times 2$ (Grammar: ABB, $\mathrm{ABC}) \times 2$ (Hemisphere: Left, Right) $\times 2$ (Region: Anterior, Posterior $) \times 2$ (Age: 7-month-old, 9-month-old) repeated-measures

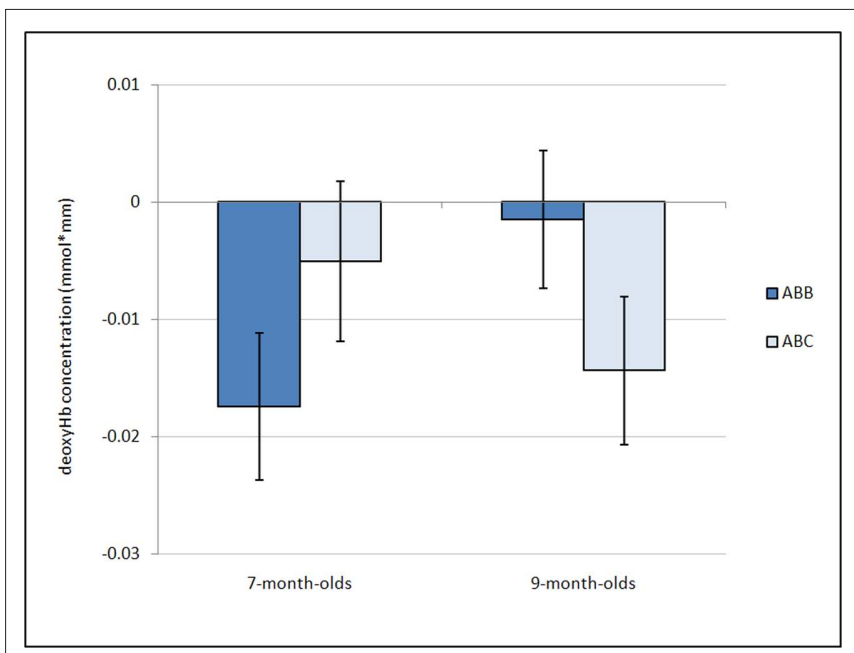

FIGURE 3 | Average deoxyHb concentration. Analysis of mean deoxy Hb concentration revealed a marginal age by condition interaction ( $p=0.08$ ). Post hoc analyses revealed that 7-month-olds show a negative response significantly different from zero for the ABB grammar, while the 9-month-olds show a negative response significantly different from zero for the ABC grammar. Error bars represent \pm SE.

ANOVA with time, grammar, hemisphere, and region as withinsubjects factors and age as the between-subjects factor revealed no main effects or interactions.

\section{DeoxyHb response across blocks}

Parallel to the analysis described above, a repeated-measures ANOVA with time, grammar, hemisphere, and region as withinsubjects factors and age as the between-subjects factor examined deoxyHb responding within the first four and last four blocks of the experiment. This analysis revealed several findings. First, as in the average deoxyHb analysis, a main effect of region was observed, $F(1,22)=19.09, p<0.001, \eta_{\mathrm{p}}^{2}=0.47$, with greater activation in anterior regions $\left(M=-0.015 \mathrm{mmol}^{*} \mathrm{~mm}, \mathrm{SD}=0.026\right)$ than posterior regions $\left(M=-0.003 \mathrm{mmol}^{*} \mathrm{~mm}, \mathrm{SD}=0.020\right)$. Additionally, there was a significant interaction between grammar and time, $F(1,22)=5.80, p=0.025, \eta_{p}^{2}=0.21$, such that the ABC grammar showed a significantly greater negative deoxyHb response during 
the first four blocks than the last four blocks, $t(23)=2.56, p=0.017$, $d=0.61$, but the ABB grammar showed no difference across time, $t(23)=0.77, p=0.45, d=0.21$. One-sample $t$-tests revealed that the $\mathrm{ABC}$ response during the first four blocks was significantly different from zero, $t(23)=-2.94, p=0.007, d=0.60$, and that the $\mathrm{ABB}$ response during the last four blocks was marginally different from zero, $t(23)=-1.83, p=0.08, d=0.37$.

Finally, a marginal interaction between grammar and age was also found, $F(1,22)=4.06, p=0.056, \eta_{p}^{2}=0.16$, with 7-montholds showing greater activation to the $\mathrm{ABB}$ grammar than the $\mathrm{ABC}$ grammar, and 9-month-olds showing greater activation to $\mathrm{ABC}$ than $\mathrm{ABB}$, though neither difference reaches significance ( $p$ s > 0.1). One-sample $t$-tests revealed that overall activation to the ABB grammar in 7-month-olds was significantly different from zero, $t(12)=-2.78, p=0.017, d=0.77$, and activation to the ABC grammar in 9-month-olds was significantly different from zero, $t(10)=-2.14, p=0.058, d=0.64$ (see Figure 4).

\section{DISCUSSION}

In the present study we examined oxyHb and deoxyHb responses to two artificial grammars, one containing syllables in an $\mathrm{ABB}$ pattern and the other containing syllables in an ABC pattern. Analyses examining the average oxyHb response to each grammar across all trials of the experiment as well as across early and late blocks found no differential responses relating to hemisphere or region and no differentiation between the $\mathrm{ABB}$ and $\mathrm{ABC}$ grammars. Across analyses examining deoxy $\mathrm{Hb}$ responding, overall greater negative response was found in anterior regions as compared to posterior regions, and 7- and 9-month-olds showed trends toward differential responding to the $\mathrm{ABB}$ and $\mathrm{ABC}$ grammars. Specifically, 7-month-olds showed deoxyHb responding significantly different from zero for the $\mathrm{ABB}$ grammar and not the $\mathrm{ABC}$ grammar,

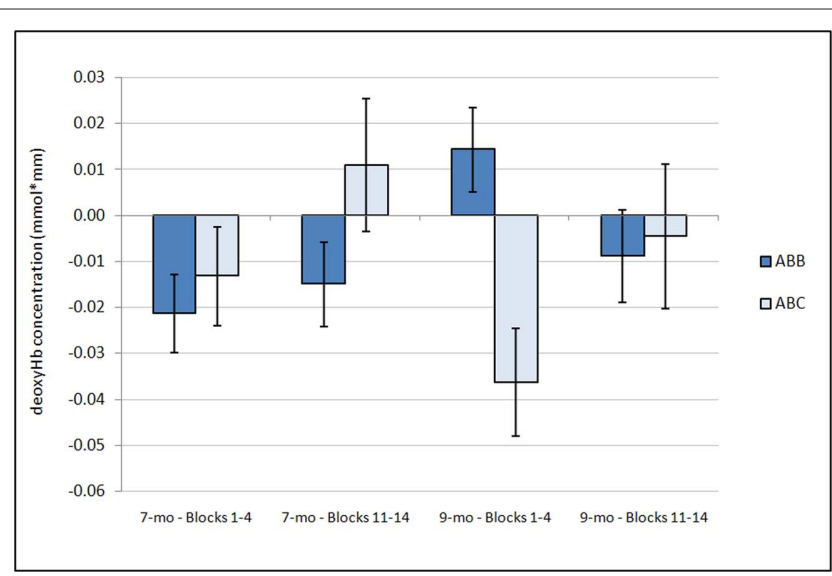

FIGURE 4 | Average deoxyHb concentration over time. Analysis of deoxyHb concentration during the first four blocks and the last four blocks revealed a significant interaction between condition and time $(p=0.025)$, with a significantly larger deoxyHb response for the $\mathrm{ABC}$ grammar during the early blocks than the later blocks $(p<0.02)$ and no change in ABB grammar responding across time $(p=0.45)$. Additionally, a marginal interaction was found between condition and age $(p=0.056)$. One-sample $t$-tests revealed that the 7-month-old ABB response during Blocks $1-4$ and the 9-month-old $A B C$ response during Blocks 1-4 are significantly different from zero. Error bars represent \pm SE. while 9-month-olds showed the opposite effect, with activation significantly different from zero for the $\mathrm{ABC}$ grammar and not the ABB grammar.

Average deoxy $\mathrm{Hb}$ across all trials also revealed an interaction between region and hemisphere, with posterior regions showing no differences in responding across hemispheres, but significantly greater activation in the right anterior region than the left anterior region. When examining deoxyHb activation across early and late blocks, a grammar by time interaction was observed as well. Between the early and late blocks, ABC activation significantly decreased, while no difference in $\mathrm{ABB}$ responding was found between the two time points. The deoxyHb response to $\mathrm{ABC}$ during the early trials was significantly different than zero.

With regards to localization of the neural response, parallel to the oxyHb findings from Gervain et al. (2008), the present study revealed stronger deoxyHb activation in anterior regions as compared to posterior regions. However, no left hemisphere bias was found, contrary to prior studies using auditory stimuli with infants from 0- to 4-months-of-age (e.g., Dehaene-Lambertz, 2000; Dehaene-Lambertz et al., 2002; Pena et al., 2003). In fact, the only significant finding relating to hemisphere in the present work identified greater deoxyHb responding in right anterior regions as compared to left anterior regions. Although the work of Gervain et al. (2008) showed no absolute left hemisphere bias to the syllables in newborns, the oxyHb response to the ABB grammar did show a significantly greater response in the left hemisphere, raising the question of why the 7- and 9-month-old infants in the present study show little influence of the left hemisphere on hemodynamic activity. In considering important changes in speech perception that occur between 8- and 12-months-of-age (e.g., Werker, 1989), it is possible that the present stimuli, spoken by a native French speaker, could mark these stimuli as less linguistically meaningful to children as they become older. Further, recent work by Homae et al. (2007) found stronger right hemisphere activation for flattened speech streams, and because the present stimuli contained monotonous prosody, this could perhaps result in right hemisphere activation that tempers any left hemisphere dominance that might otherwise be found relating to rule learning and pattern extraction processes.

By using NIRS to examine the neural response to $\mathrm{ABB}$ and $\mathrm{ABC}$ grammars in 7- and 9-month-old infants, the present work extends the newborn ABB and ABC study of Gervain et al. (2008). Gervain et al. (2008) found greater oxyHb activation in response to the $\mathrm{ABB}$ grammar than the $\mathrm{ABC}$ grammar, and this was more pronounced in the anterior regions of the newborn brain. The current study found a developmental shift in this response, with 7-month-old infants showing a significant response to $\mathrm{ABB}$ blocks but not $\mathrm{ABC}$ blocks, and 9-month-olds showing a significant response to $\mathrm{ABC}$ blocks but not $\mathrm{ABB}$ blocks. The heightened neural attunement to differing patterns at different ages is consistent with behavioral work illustrating shifts in sensitivity to repetition patterns during the first year of life (e.g., Johnson et al., 2009).

With larger negative deoxyHb responses expected to correspond with greater activation to that stimulus (see Obrig et al., 2010 , for a review), the present findings suggest changes in the salience of the $\mathrm{ABB}$ and $\mathrm{ABC}$ grammars across the first year of life, as newborns (Gervain et al., 2008) and 7-month-olds show more negative deoxyHb responding to the $\mathrm{ABB}$ grammar and 
9-month-olds show more negative responding to the $\mathrm{ABC}$ grammar This shift in salience from ABB to ABC over the first year of life might parallel infant looking-time findings that show that, after a familiarization period, preference for the familiar stimulus is found in younger infants, while preference for the novel stimulus can be found in older infants (e.g., Ferry et al., 2010). Aslin (2007) describes this difference in terms of a balance, whereby "...stimuli can trigger a recognition response for familiarity that is stronger than the attentional response to novelty." The newborns tested by Gervain et al. (2008) could be neurally attuned to the familiarity and regularity of the repetition rule within the ABB blocks, while the ABC grammar could represent an overly complex stimulus to 1-day-old participants. On the other hand, in 7- to 9-months-olds who have been exposed to a wider range of auditory input and can proficiently extract regularities from the surrounding speech stream (e.g., Saffran et al., 1996), it is possible that the easily detectable repetition in ABB blocks is redundant and requires less neural processing than the consistently novel ABC input.

Gervain et al. (2008) found that over the course of their experiment, newborns showed an interaction between grammar and time, such that the $\mathrm{ABB}$ grammar showed increasing activation from the early blocks to the late blocks, while the ABC grammar showed no change. The present study also found an interaction between these two variables: 7- to 9-month-olds show a different response pattern, with significantly more activation to $\mathrm{ABC}$ during the first four blocks as compared to the last four blocks, and no difference between $\mathrm{ABB}$ activation across time. In the newborn study, infants become increasingly responsive to their preferred stimulus throughout the experiment, while 7- to 9-month-olds showed responding significantly greater than zero for only the ABC stimulus early on, but no significant difference from zero for either stimulus during late blocks. One probable explanation for this early differentiation followed by no differentiation in the 7- to 9-month-olds is that the experiment extended beyond their interest and led to diminished attention and responding to the differences between the $\mathrm{ABB}$ and $\mathrm{ABC}$ grammars. A recent review by Turk-Browne et al. (2008) discusses infant studies that resulted in either the habituation of responses across time or the enhancement of responses across time and the related phenomenon in adult neuroimaging work, but the factors that lead to habituation vs. enhancement remain unclear. In order to further delineate developmental shifts between the repetition enhancement found by Gervain et al. (2008) in newborns and the repetition suppression found in the present study with older infants, work is under way using the present auditory NIRS task with a wider age range of infants.

Finally, it is important to mention that while Gervain et al. (2008) found significant effects in their analyses of oxyHb activation, in the present study we only find effects in analyses of deoxyHb activation. Cortical activation is traditionally thought to result in both an increase in oxy $\mathrm{Hb}$ and a decrease in deoxyHb; however, studies using NIRS often find stronger effects in one over the other. Many complex factors likely contribute to how robustly oxy- and deoxyHb activation are coupled in infant NIRS experiments, including the equipment used, the ages tested, and the areas of the brain examined (see Gervain et al., 2011, for further discussion). While many infant NIRS studies have reported significant oxyHb effects alongside few or no significant deoxy $\mathrm{Hb}$ effects (e.g., Saito et al., 2007a,b; Gervain et al., 2008; Lloyd-Fox et al., 2009; Sato et al., 2009), some studies have reported significant activation in both oxyHb and deoxyHb (e.g., Wilcox et al., 2005; Homae et al., 2006). The first year of life includes developmental changes in brain maturation such as changes in vascular tone and blood flow patterns, and NIRS work should continue to explore how this development differentially affects oxyHb and deoxyHb responding. The importance of understanding changes in deoxyHb has been emphasized by adult neuroimaging work that identifies deoxyHb as the species of hemoglobin correlated with the blood oxygenation level-dependent response found in fMRI studies (e.g., Kleinschmidt et al., 1996; see Steinbrink et al., 2006, for a review). This fMRI/NIRS literature supports data interpretation that gives at least as much weight to deoxyHb findings as oxyHb findings, an important consideration for future infant NIRS studies.

An important limitation of the present study is the varying attentional state of the infant participants throughout the session due to the range of silent activities permitted to keep them free of motion (e.g., watching videos, examining novel toys, drinking from a bottle, sleeping). Because of the more limited time span for keeping 7- and 9-month-old infants still during this 15-min task as compared to the newborn infants tested in Gervain et al. (2008), follow-up studies in older infants should consider a paradigm with fewer blocks of shorter duration in order to equate testing conditions across infants as carefully as possible.

\section{CONCLUSION}

In summary, the present work has identified bilateral regions in 7- and 9-month-old infants that show neural attunement to blocks of syllables containing a repetition $(\mathrm{ABB})$ and blocks without a repetition (ABC). While newborn infants (Gervain et al., 2008) and 7-month-old infants showed heightened activity to ABB stimuli, 9-month-old infants showed greater responding to the ABC grammar, perhaps reflecting increased attention to the complexity of the always-changing stimulus. Current work with 3- and 12-month-old infants is underway to more clearly delineate changes in activation across the first year of life to stimuli with and without structural regularities. Specifically, this work will explore the shifting contributions of both oxyHb and deoxyHb in differentiating linguistically relevant information and contribute to our understanding of the factors influencing hemispheric lateralization in response to auditory stimuli. Moreover, this work will allow researchers to gain further insight into the neural bases of developmental shifts in abstract rule learning.

\section{ACKNOWLEDGMENTS}

This research was made possible, in part, by grants from the Simons Foundation and the NIDCD (R21 DC 08637 \& R01 DC 10290), from the NIMH-funded Clinical Research Training Program at Harvard Medical School (to Jennifer B. Wagner), and from the Hugh Hampton Young Memorial Foundation \& the NIH-funded Neuroimaging Training Program (to Sharon E. Fox). 


\section{REFERENCES}

Aslin, R. N. (2007). What's in a look? Dev. Sci. 10, 48-53.

Christophe, A., Dupoux, E., Bertoncini, J., and Mehler,J.(1994). Do infants perceive word boundaries? An empirical study of the bootstrapping of lexical acquisition. J. Acoust. Soc. Am. 95, 1570-1580.

Dawson, C., and Gerken, L. (2009). From domain-generality to domainsensitivity: 4-month-olds learn an abstract repetition rule in music that 7-month-olds do not. Cognition 111, 378-382.

Dehaene-Lambertz, G. (2000). Cerebral specialization for speech and nonspeech stimuli in infants. J. Cogn. Neurosci. 12, 449-460.

Dehaene-Lambertz, G., Dehaene, S., and Hertz-Pannier, L. (2002). Functional neuroimaging of speech perception in infants. Science 298, 2013-2015.

Ferry, A. L., Hespos, S. J., and Waxman, S. R. (2010). Categorization in 3- and 4-month-old infants: an advantage of words over tones. Child Dev. 81, 472-479.

Frank, M. C., Slemmer, J.A., Marcus, G. F., and Johnson, S. P. (2009). Information from multiple modalities helps 5-month-olds learn abstract rules. Dev. Sci. 12, 504-509.

Gervain, J., Macagno, F., Cogoi, S., Pena, M., and Mehler,J. (2008). The neonate brain detects speech structure. Proc. Natl. Acad. Sci. U.S.A. 105, 14222-14227.

Gervain, J., and Mehler, J. (2010). Speech perception and language acquisition in the first year of life. Annu. Rev. Psychol. 61, 191-218.

Gervain, J., Mehler, J., Werker, J. F., Nelson, C. A., Csibra, G., Lloyd-Fox, S., Shukla, M., and Aslin, R. N. (2011). Nearinfrared spectroscopy: a report from the McDonnell infant methodology consortium. Dev. Cogn. Neurosci. 1, 22-46.

Homae, F., Watanabe, H., Nakano, T., Asakawa, K., and Taga, G. (2006). The right hemisphere of sleeping infant perceives sentential prosody. Neurosci. Res. 54, 276-280.

Homae, F., Watanabe, H., Nakano, T., and Taga, G. (2007). Prosodic processing in the developing brain. Neurosci. Res. 59, 29-39.

Johnson, S. P., Fernandas, K. J., Frank, M. C., Kirkham, N., Marcus, G., Rabagliati, H., and Slemmer, J. A. (2009). Abstract rule learning for visual sequences in 8 - and 11-montholds. Infancy 14, 2-18.

Kleinschmidt, A., Obrig, H., Requardt, M. Merboldt, K., Dirnagl, U., VIllringer, A., and Frahm, J. (1996). Simultaneous recording of cerebral blood oxygenation changes during human brain activation by magnetic resonance imaging and near-infrared spectroscopy. $J$. Cereb. Blood Flow Metab. 16, 817-826.

Kuhl, P. K., Stevens, E., Hayashi, A., Deguchi, T., Kiritani, S., and Iverson, P. (2006). Infants show a facilitation effect for native language phonetic perception between 6 and 12 months. Dev. Sci. 9, F13-F21.

Little, V. M., Thomas, D. G., and Letterman, M. R. (1999). Single-trial analyses of developmental trends in infant auditory event-related potentials. Dev. Neuropsychol. 16, 455-478.

Lloyd-Fox, S., Blasi,A., Volein,A., Everdell, N., Elwell, C. E., and Johnson, M. H. (2009). Social perception in infancy: a near infrared spectroscopy study. Child Dev. 80, 986-999.

Marcus, G., Fernandes, K., and Johnson, S. (2007). Infant rule-learning facilitated by speech. Psychol. Sci. 18, 387-391.

Marcus, G., Vijayan, S., Bandi Rao, S., and Vishton, P. M. (1999). Rule-learning in seven-month-old infants. Science 283, 77-80.

Mehler, J., Jusczyk, P., Lambertz, G., Halsted, N., Bertoncini, J., and AmielTison, C. (1988). A precursor of language acquisition in young infants. Cognition 29, 143-178.

Minagawa-Kawai, Y., Mori, K., Naoi, N., and Kojima, S. (2007). Neural attunement processes in infants during the acquisition of a language-specific phonemic contrast. J. Neurosci. 27, 315-321.

Obrig, H., Rossi, S., Telkemeyer, S., and Wartenburger, I. (2010). From acoustic segmentation to language processing: evidence from optical imaging. Front. Neuroenergetics 2:13. doi: 10.3389/fnene.2010.00013

Pena, M., Maki, A., Kovacic, D., DehaeneLambertz, G., Koizumi, H., Bouquet, F., and Mehler, J. (2003). Sounds and silence: an optical topography study of language recognition at birth. Proc. Natl. Acad. Sci. U.S.A. 100 11702-11705.

Saffran, J. R., Aslin, R. N., and Newport, E. L. (1996). Statistical learning by 8-month-old infants. Science 274, 1926-1928.

Saffran, J. R., Pollack, S. D., Seibel, R. L. and Shkolnik, A. (2007). Dog is a dog is a dog: infant rule learning is not specific to language. Cognition 105, 669-680.

Saito, Y., Aoyama, S., Kondo, T. Fukumoto, R., Konishi, N., Nakamura, K., Kobayashi, M., and Toshima, T. (2007a). Frontal cerebral blood flow change associated with infant-directed speech. Arch. Dis. Child. Fetal Neonatal Ed. 92, F113-F116.

Saito, Y., Kondo, T., Aoyama, S., Fukumoto, R., Konishi, N., Nakamura, K., Kobayashi, M., and Toshima, T. (2007b). The function of the fronta lobe in neonates for response to a prosodic voice. Early Hum. Dev. 83 225-230.

Sato, Y., Sogabe, Y., and Mazuka, R. (2009). Development of hemispheric specialization for lexical pitch-accent in Japanese infants. J. Cogn. Neurosci. 22, 2503-2513.

Steinbrink, J., Villringer, A., Kempf, F. Haux, D., Boden, S., and Obrig, $\mathrm{H}$. (2006). Illuminating the BOLD signal: combined fMRI-fNIRS studies. Magn Reson. Imaging 24, 495-505.

Teinonen, T., Fellman, V., Naatanen, R., Alku, P., and Huotilainen, M. (2009). Statistical language learning in neonates revealed by event-related brain potentials. BMC Neurosci. 10, 21. doi: 10.1186/1471-2202-10-21

Telkemeyer, S., Rossi, S., Koch, S. P., Nierhaus, T., Steinbrink, J., Poeppel, D., Obrig, H., and Wartenburger, I (2009). Sensitivity of newborn audi- tory cortex to the temporal structure of sounds. J. Neurosci. 29, 14726-14733.

Thomas, D. G., and Lykins, M. S. (1995). Event-related potential measures of 24-hour retention in 5-month-old infants. Dev. Psychol. 31, 946-957.

Turk-Browne, N. B., Scholl, B. J., and Chun, M. M. (2008). Babies and brains: habituation in infant cognition and functional neuroimaging. Front. Hum. Neurosci. 2:16. doi: 10.3389/ neuro.09.016.2008

Werker, J. F. (1989). Becoming a native listner. Am. Sci. 77, 54-69.

Werker, J. F., and Tees, R. C. (1984). Crosslanguage speech perception: evidence for perceptual reorganization during the first year of life. Infant Behav. Dev. 7, 49-63.

Wilcox, T., Bortfeld, H., Woods, R., Wruck, E., and Boas, D. A. (2005). Using near-infrared spectroscopy to assess neural activation during object processing in infants. J. Biomed. Opt. 10, 11010-11026.

Conflict of Interest Statement: The authors declare that the research was conducted in the absence of any commercial or financial relationships that could be construed as a potential conflict of interest.

Received: 15 January 2011; accepted: 05 July 2011; published online: 25 July 2011. Citation: Wagner JB, Fox SE, TagerFlusberg H and Nelson CA (2011) Neural processing of repetition and non-repetition grammars in 7-and 9-month-old infants. Front. Psychology 2:168. doi: 10.3389/ fpsyg.2011.00168

This article was submitted to Frontiers in Language Sciences, a specialty of Frontiers in Psychology.

Copyright (C) 2011 Wagner, Fox, TagerFlusberg and Nelson. This is an openaccess article subject to a non-exclusive license between the authors and Frontier Media SA, which permits use, distribution and reproduction in other forums, provided the original authors and source are credited and other Frontiers conditions are complied with. 


\section{APPENDIX}

Statistical maps ( $t$-maps) comparing oxyHb and deoxyHb responses to the repetition $(\mathrm{ABB})$ and non-repetition $(\mathrm{ABC})$ grammars for 7-month-olds (Figure A1) and 9-month-olds (Figure A2). For $A B B$ vs. $A B C$ in oxyHb $t$-tests (left side of Figures $A 1$ and $A 2$ ), warmer colors (reds) signify greater activation to ABB grammar as compared to $\mathrm{ABC}$ grammar; for deoxyHb $t$-tests (right side of Figures A1 and A2), colder colors (blues) signify greater activation to $\mathrm{ABB}$ as compared to $\mathrm{ABC}$. No channels showed significant differences between the two grammars (all ps $>0.05$ ).



FIGURE A1 | Channel-by-channel $t$-tests for 7-month-old infants in the present study.

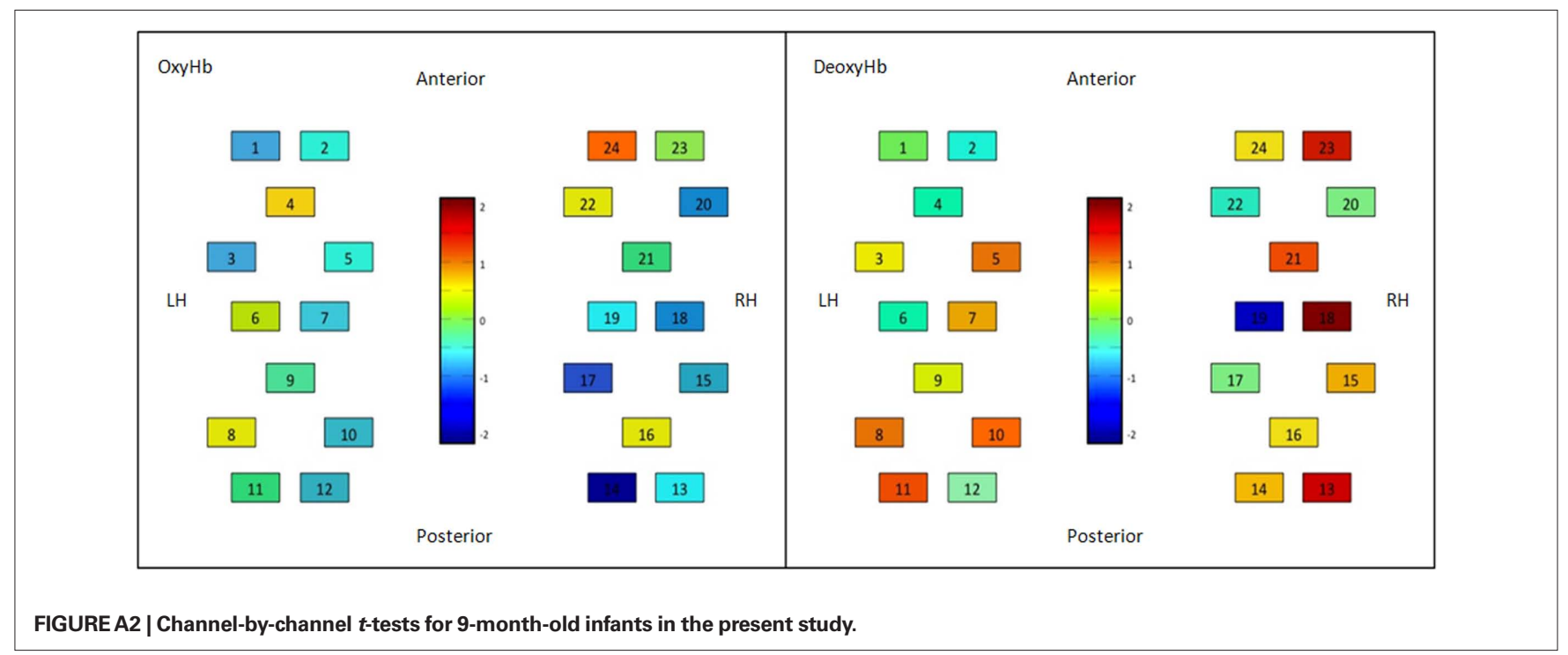

